\title{
The Study of Running Behavior of Pedestrian at Urban Signalized Intersection
}

\author{
Zhiyin Lia, Ziyang Wang ${ }^{b}$, Boran Wan, Mingtao Wu, Haiyue Zhao \\ School of Traffic and Transportation, Beijing Jiaotong University, Beijing 100044, China \\ a14120950@bjtu.edu.cn, bwangzy@bjtu.edu.cn
}

Keywords: signalized intersection, running pedestrian, relevance, regression analysis, survival analysis.

\begin{abstract}
Pedestrian is the main traffic participants in the signalized intersection. And its running behavior, on the one hand, will increase hidden danger because of reducing driver's reaction time; on the other hand, it will decrease the time of exposure to the road and reduce the security risk. The study found that the occurrence of running behavior has very obvious regularity with the time of the signal lights began. Through the video observation, we study on 268 available samples that start running at the green and yellow light with correlation analysis, survival analysis and multivariate linear regression model. It was found that the running behavior occurred regularly with the time of the signal lights and finally got the regression model. Among pedestrian-vehicle conflict, most of the sample started running at the beginning of the green light time occurred in the case of the vehicles not yield to the pedestrian. The sample waiting longer time before the start of running always start at a long distance to the end of green light. These findings can be applied to the design of intelligent transportation systems and can also provide data and theoretical support for the further study.
\end{abstract}

\section{Introduction}

Pedestrian are traffic participants out of the vehicle and no protection, and vulnerable road users in the world. They are easy to get hurt in the traffic accident, even serious injury or death. In China, $40 \%$ of the trips are completed by walking [1], exposing many pedestrians to danger. In 2007, 21106 pedestrian deaths and 70838 injuries happened, making respectively $25.85 \%$ and $18.62 \%$ of the total deaths and injuries on the road [2]. Except for some nature disasters, these accidents are all caused by human factors [3]. Pedestrian safety is always a very significant part of traffic safety.

Urban transportation system is one of the basic components of human activities while signalized intersection is a very important part of urban transportation system and a typical road condition. Pedestrian behavior is the main reason that causes the disorder of traffic order, low efficiency and hidden danger at the signalized intersection. There are many researches about the pedestrian behaviors in previous studies. Changxu $\mathrm{Wu}$ et al. research the red-light running behavior of electric bike and cyclists at urban intersections [4]; Xiangling Zhuang et al. study the safety margin and perceived safety of pedestrians at unmarked roadway [5]; Haichan Wang studied the red-light behavior of pedestrian based on the survival analysis method [6]. Somebody else research the behavior of pedestrians through virtual experiments at signalized intersection [7]. Zhiyong Sun et al. study the acceptance choice of pedestrians in the process of crossing the signalized intersection [8].

The study has found that running crossing the intersection will increase hidden danger because of reducing driver's reaction time and decrease the time of exposure to the road which can reduce the security risk $[9,10]$ at the same time, which make the running behavior a very worthwhile study. However, there are very few studies focusing on the running behavior. It was just used as an index of risky behavior [11,12]. And there is no further research on it. The purpose of this paper is to discuss the distribution characteristics of running crossing behavior of the pedestrian at the signalized intersection, correlative factors and the relevance between the factors. Finally we can get the model of related factors, which can give reasonable suggestions to the drivers and pedestrians, put forward the 
improvement measures for the road facilities, and provide data and theoretical support for the further study.

\section{Methods}

Field observations with video recording were used in this study. They have been widely used to investigate of traffic environment [13]. This approach is also used to carry out the study of path effects [14].

\subsection{Observation Location}

A typical signalized intersection was chosen at Wangfujing area in Beijing City, China. One side is the shopping center while the other side is the snack break area. This intersection is a double lane, very busy with 624 vehicles and 3986 pedestrians per hour just the crosswalk observed.

\subsection{Observation and coding}

A camera was set on the building besides the intersection on two temperate days, namely 11AM until 12:20 at noon on March 7th, PM 1:00 4:00 on March 15th and 10 AM to 13 PM at afternoon on March 21th. Expand the vision as far as possible on the basis of pedestrians can be observed on the details, to see the whole picture of the crosswalk without being blocked. The whole shooting process is carried out in the case of no traffic assist manager to obtain the nature behavior of pedestrians.

Table 1 Variables Considered in Study

\begin{tabular}{|c|c|c|}
\hline Aspect & Variable & Description \\
\hline \multirow{4}{*}{$\begin{array}{l}\text { Demographic } \\
\text { aspect }\end{array}$} & gender & Male/ Female \\
\hline & age & Young: 0 35years old; Middle-age: older than 35 years old \\
\hline & group_size & the total number of people on the sidewalk at the start of the running \\
\hline & conflict & Pedestrian-vehicle conflict: Yes / no \\
\hline \multirow[t]{5}{*}{$\begin{array}{l}\text { Context related } \\
\text { aspect }\end{array}$} & vehi_yield & $\begin{array}{l}\text { in the case of pedestrian-vehicle conflict, whether the vehicle yield } \\
\text { to the pedestrian or not }\end{array}$ \\
\hline & vehi_type & $\begin{array}{l}\text { the type of the vehicle in the case of pedestrian-vehicle conflict: Car/ } \\
\text { Bus }\end{array}$ \\
\hline & delta-T & the time difference of beginning running and the end of yellow light \\
\hline & path_style & path type for running across the road: 4 types \\
\hline & look & $\begin{array}{l}\text { before and during cross the road, whether look around: judge by } \\
\text { head swing: Yes/ No }\end{array}$ \\
\hline \multirow[t]{2}{*}{$\begin{array}{l}\text { Behavioral } \\
\text { aspect }\end{array}$} & $\begin{array}{l}\text { stop } \\
\text { sideWait_time } \\
\text { beforeWait_time } \\
\text { running_time }\end{array}$ & $\begin{array}{c}\text { whether stop before running or on the roadside: Yes/ No } \\
\text { the time pedestrian waited on the roadside before cross the road } \\
\text { the time pedestrian waited before running } \\
\text { running time }\end{array}$ \\
\hline & $\begin{array}{l}\text { before_crosswalk } \\
\text { after_crosswalk }\end{array}$ & $\begin{array}{l}\text { began to run before entering the sidewalk: Yes/ No } \\
\text { still run after leaving the sidewalk: Yes/ No }\end{array}$ \\
\hline
\end{tabular}

The video for a total of 7 hours and 20 minutes recorded 566 pedestrians with running behavior. Remove the pedestrians that cannot be seen clearly at any time in the whole running process, 492 samples remaining, including 268 samples started during the green and 224 samples started during red light. In this study, only the samples that started during the green were taken as the research objects. The signal lamp of the intersection observed has 50s for green in each cycle.

Most variables could be coded with the description in Table I. Further remarks are needed for some variables. The "age" was coded into two groups: "Young" refers to the people in the range of $0 \sim 35$ years and "Middle-age" represent the people older than 35 years because the children in the age of 0 $\sim 16$ years and the elderly older than 60 are very few. In the phenomenon of pedestrian- vehicle conflict, we determine whether the vehicle yield or not by obvious deceleration behavior of the vehicle. For the behavior looking at vehicles, a noticeable turn of head is needed. Path types are mainly divided into 4 types, as shown in Figure1. In particular, to facilitate statistics and description, we do not distinguish between green and yellow light in the following paper, so the "delta- $T$ " refer to the time to the end of the yellow light at the beginning of running. 


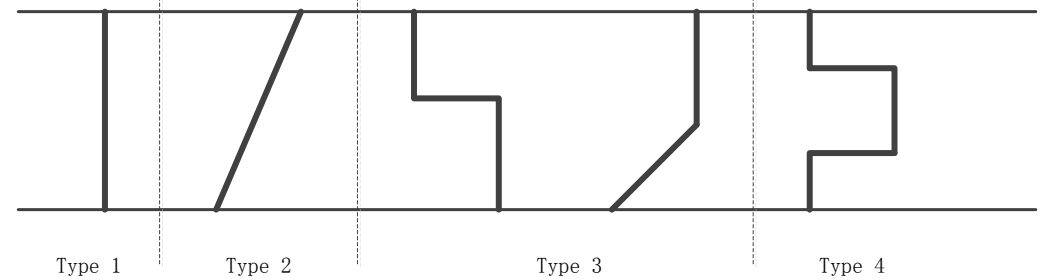

Fig. 1 Path type

\section{Results}

\subsection{Statistical and Description Analysis}

Statistical description of the 268 samples is shown in Table 2. From the demographic aspects, male were more than female (Female: 39.18\%; Male: 60.82\%); young runners are more than middle-aged runners (Young: 61.19\%; Middle-age: 38.81\%). As context aspect, there were 32.28 pedestrians on the crosswalk for each sample on average, which reflects that the runner was in a relatively large group. The conflict between people and vehicles accounted for $43.66 \%$, which is very detrimental to the normal traffic and $76.92 \%$ of the conflict was happened between car and pedestrians and in the conflict, $88.03 \%$ of vehicles had the yielding behavior. From the behavioral aspect, there were $61.94 \%$ of the samples with the behavior of looking around; as the path type, $51.12 \%$ of the runners choose the type 1 and $36.94 \%$ choose the type 2 while only $11.94 \%$ of the samples choose the type 3 and type 4; only 17 samples stop at the roadside or before running. The average waiting time is $0.44 \mathrm{~s}$ before the start of running and it is $0.67 \mathrm{~s}$ waiting at the roadside. The average running time of the sample was 3.05s. Most running behavior occurred inside the crosswalk, while only $28.73 \%$ of the runners started running before entering the sidewalk and $10.82 \%$ still ran after leaving the sidewalk.

Table 2 Statistics of Sample Variables

\begin{tabular}{cccccccc}
\hline Variable & Level & N & Variable & Level & N & Variable & Mean value \\
\hline \multirow{2}{*}{ gender } & Female & 105 & \multirow{2}{*}{ before_crosswalk } & Yes & 77 & group_size & 32.28 \\
& Male & 163 & & No & 191 & sideWait_time & 0.44 \\
age & Young & 164 & after_crosswalk & Yes & 29 & beforeWait_time & 0.67 \\
& Middle-age & 104 & & No & 239 & running_time & 3.05 \\
look & Yes & 166 & conflict & Yes & 117 & & \\
& No & 102 & & No & 151 & & \\
& 1 & 137 & vehi_yield & Yes & 103 & & \\
path_style & 2 & 99 & (conflict) & No & 14 & & \\
& 3 & 22 & vehi_type & Car & 90 & & \\
& 4 & 10 & (conflict) & Bus & 27 & & \\
stop & Yes & 17 & & & & & \\
& No & 251 & & & & & \\
& & & & & & & \\
& & & & & & & \\
\end{tabular}

The relationship between the end time of the green light and the sample distribution is shown in Figure2. It can be seen that most running phenomena occurred at the end of the green light. In the end of $5 \mathrm{~s}$, the samples accounted for $31.3 \%$; in the end of $10 \mathrm{~s}$, the runners accounted for $48.5 \%$. Within the first $30 \mathrm{~s}$ of the start of the green light, there only were $29.1 \%$ of the samples. Besides, at the beginning of the green light within 20s, the number of running people was in a downward trend. The number of the samples started running at the first $5 \mathrm{~s}$ accounted for $46.43 \%$ of the samples started running at the first 20s.of the green light. With the delta-T to the end of the green light less, many pedestrians worried about not being successfully through the road during the green light; at the beginning of green light, some of the pedestrians ran cross the road because of waiting too long during the red light before. 


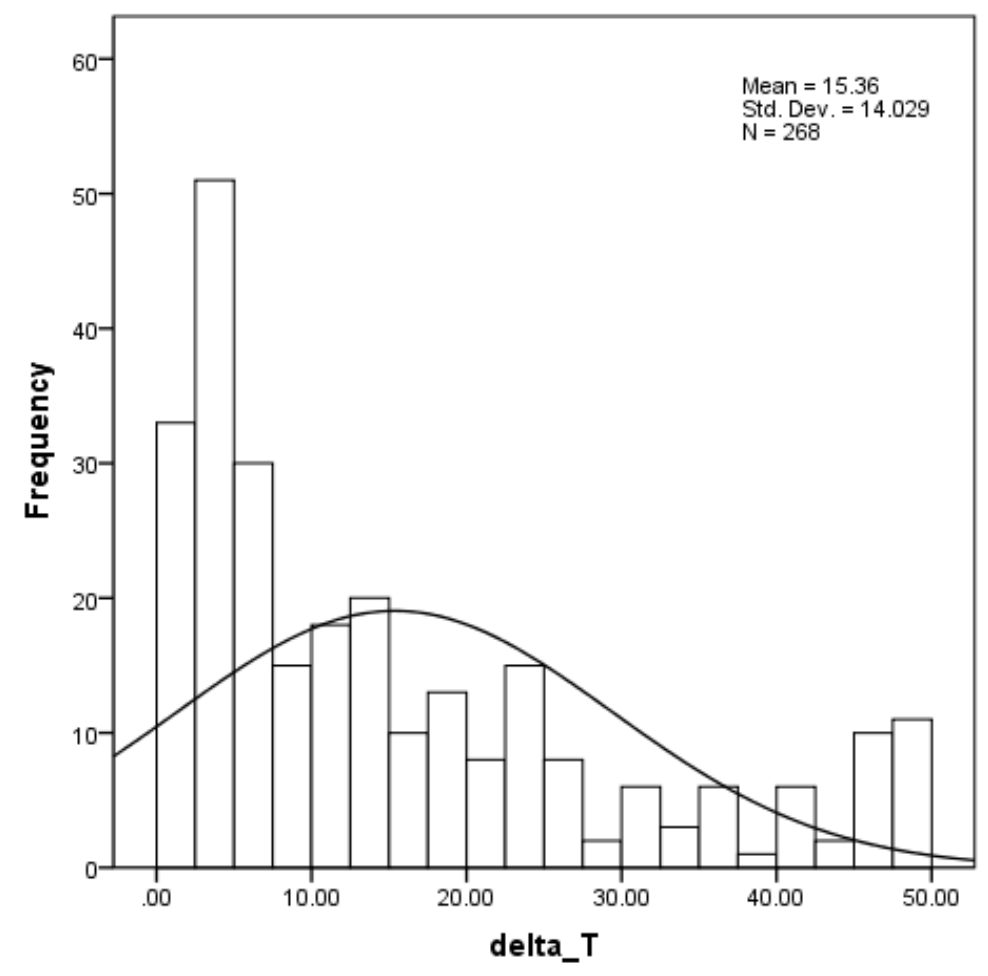

Fig. 2 Sample distribution with green light time

\subsection{Correlation Analysis}

By analysis the relationship between the variables and delta-T, we find 9 variables correlated with it: age $(\mathrm{r}=.126, \mathrm{p}<.05)$, running_time $(\mathrm{r}=-.128, \mathrm{p}<.05)$, conflict $(\mathrm{r}=.187, \mathrm{p}<.01)$, vehi_yield $(\mathrm{r}=$ $-.276, \mathrm{p}<.01)$, stop $(\mathrm{r}=.279, \mathrm{p}<.01)$, beforeWait_time $(\mathrm{r}=.263, \mathrm{p}<.01)$, sideWait_time $(\mathrm{r}=.305, \mathrm{p}$ $<.01)$, before_crosswalk $(\mathrm{r}=-.128, \mathrm{p}<.05)$, after_crosswalk $(\mathrm{r}=.226, \mathrm{p}<.01)$. The older the samples were, the longer the time was from the end of the green. The closer to the end of the green light, the longer the time of running lasted, the less running behavior caused by pedestrian-vehicle conflict and the more obvious the vehicle yielded to the pedestrians. With the beginning time of the green light, less and less samples stop, the time of the samples waiting on the roadside or before running became shorter and the behavior of starting running before entering the sidewalk or still running after leaving the crosswalk became more obvious.

Other variables are also closely related. In the samples that still ran after leaving the sidewalk, the women were more than men $(\mathrm{r}=-.237, \mathrm{p}<.01)$. If the running behavior was caused by pedestrian-vehicle conflict, the running time would be shorter $(r=-.223, p<.01)$, because these samples tend to stop running after bypassing vehicles; the pedestrians would tend to choose the path type 3 and type $4(\mathrm{r}=.215, \mathrm{p}<.01)$, because of the existence of the vehicles, pedestrians must bypass it; the behavior of looking around was more obvious $(r=.396, p<.01)$, since the pedestrians would be more careful in the case of pedestrian-vehicle conflict; less samples would start running before enter the crosswalk $(\mathrm{r}=-.359, \mathrm{p}<.01)$ or still run after leaving the sidewalk $(\mathrm{r}=-.186, \mathrm{p}<.01)$, the samples started running before enter the sidewalk were mostly to get the green light while the runner still ran after leaving the crosswalk were mostly in a hurry instead of a conflict. On the contrary, the running time was relatively long if the vehicle yield to the pedestrian in the conflict $(r=.201, p<.05)$, because the runner would ran faster in the case that the vehicle did not yield to the pedestrian. Car rather than bus easier yielding to pedestrians in the conflict $(r=-.298, p<.01)$, it is due to the fear of the pedestrian to a big vehicle. The pedestrians with stopping behavior were more obvious with the behavior of looking around $(\mathrm{r}=.204, \mathrm{p}<.01)$, and the latter would wait longer time on the roadside $(\mathrm{r}$ $=.594, \mathrm{p}<.01)$ or before running $(\mathrm{r}=.441, \mathrm{p}<.01)$, this is related to the patience and caution of this part of the samples. The longer the waiting time was on the roadside, the longer the waiting time before running $(r=.667, p<.01)$. 


\subsection{Multivariate Linear Regression Model and Survival Analysis}

Based on the results of correlation analysis, stepwise regression was conducted to get the variables closely related to the delta-T to the end of green light. The entering probability is selected as 0.05 and the deletion probability is selected as 0.10 . The results are shown in Table 3 .

Table 3 Result of Regression Analysis

\begin{tabular}{|c|c|c|c|c|c|}
\hline & \multirow{2}{*}{ Model } & \multicolumn{3}{|c|}{ Unstandardized CoefficientsStandardized Coefficients } & \multirow{2}{*}{ Sig } \\
\hline & & $\mathrm{B}$ & Std. Error & Beta & \\
\hline \multirow{3}{*}{1} & (Constant) & 29.634 & 3.772 & & 7.857 .000 \\
\hline & vehi_yield & -12.360 & 4.020 & -.276 & -3.075 .003 \\
\hline & (Constant) & 29.282 & 3.617 & & 8.095 .000 \\
\hline \multirow{2}{*}{$\begin{array}{l}2 \\
\text { be }\end{array}$} & vehi_yield & -13.157 & 3.861 & -.293 & -3.407 .001 \\
\hline & eforeWait_time & 1.644 & .493 & .287 & 3.336 .00 \\
\hline
\end{tabular}

It can be seen that only vehi_yield and beforeWait_time closely related to the delta-T to the end of the green and we can get the multiple linear regression model as follows:

$$
\mathrm{y}=29.282-13.157 x_{1}+1.644 x_{2}
$$

In the formula, $\mathrm{y}$ refers to delta- $\mathrm{T}$, while $\mathrm{x}_{1}$ refers to whether the vehicle yield to the pedestrians and $\mathrm{x}_{2}$ refers to the time before the sample running.

Survival analysis was conducted for the two variables got by the regression analysis.

Figure 3 shows the survival function of the two groups of samples divided by whether or not the vehicle yielding to the pedestrian in the conflict. At the end of $5 \mathrm{~S}$ from the green light, there is no difference between the two groups of the samples. The cumulative survival function has a sharp decline at the first $3 \mathrm{~s}$ of the green light in the samples that the vehicle did not yield to in the conflict. Because there still were some vehicles rested after the red light in that period of time, which hurried though the road and were not likely to yield to pedestrians.

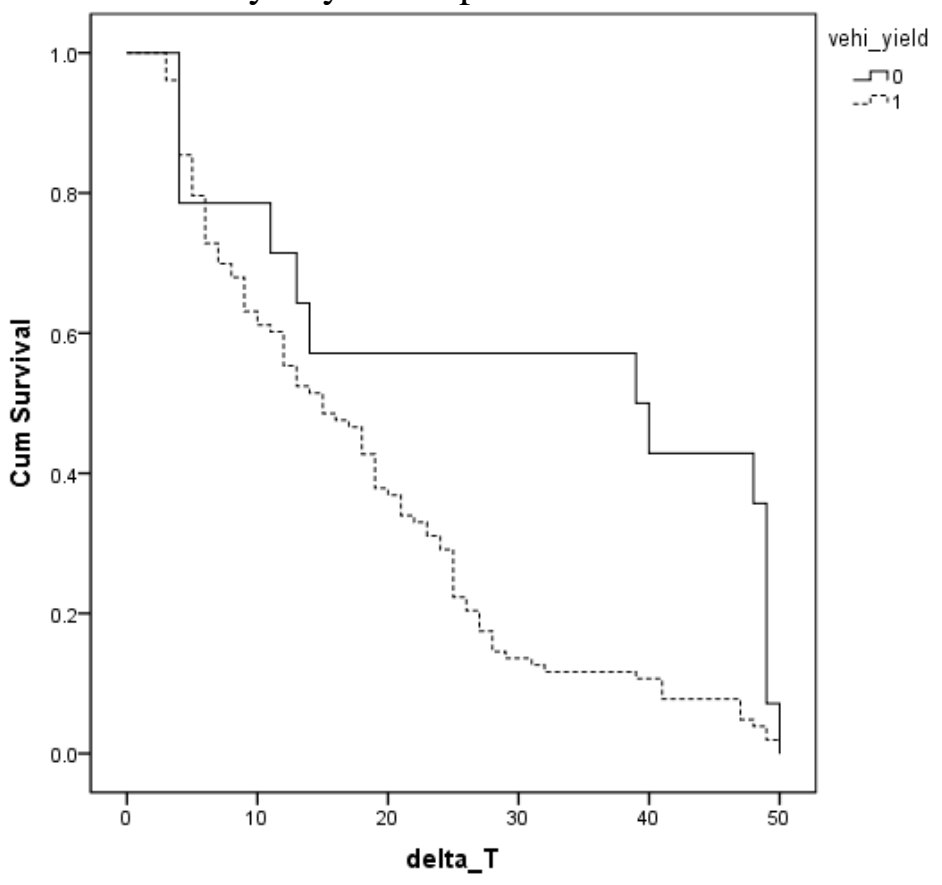

Fig. 3. The survival function of the variable of vehi_yield 


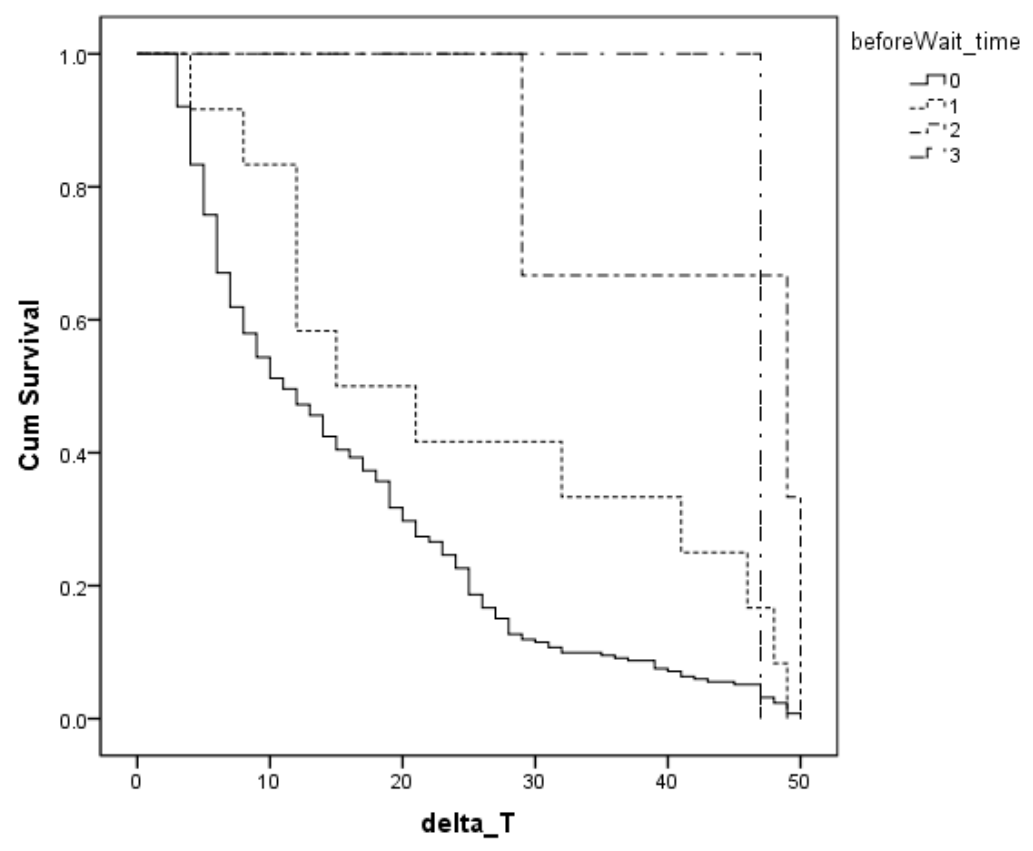

Fig. 4. The survival function of the variable of beforeWait_time

It is the survival function of the waiting time before the start of running shown in the Figure 4. In the analysis process, we define the samples without waiting, waiting for 1 10s, 11 20s, more than 20 s as $0,1,2,3$ in turn. It can be seen from the figure that the samples waiting for a long time tended to run earlier. Since at the beginning of the green light the green time is enough to pass the road, and with the green time got less and less, due to the fear of failure to pass the road during the green light time the waiting time became shorter.

\section{Discussion}

A typical signalized intersection was chosen at Wangfujing area in Beijing City, China. Field observations with video recording were used in this study and 268 pedestrians who pass the road by running were recorded. And statistical descriptive analysis, correlation analysis, stepwise regression analysis and survival analysis were carried out in turn.

\subsection{Conclusion}

Most running phenomena occurred at the end of the green light. In the end of $10 \mathrm{~s}$, the runners accounted for $48.5 \%$. Within the first 30 s of the start of the green light, there only was $29.1 \%$ of the sample. Besides, at the beginning of the green light within the $20 \mathrm{~s}$, the number of running people was in a downward trend. The number of the samples stared running at the first $5 \mathrm{~s}$ accounted for $46.43 \%$ of the samples started running at the first 20 s.

By correlation analysis the relationship between the variables and delta-T, we find 9 variables correlated with it: The older the samples were, the longer the time was from the end of the green. The closer to the end of the green light, the longer the time of running lasted, the less running behavior caused by pedestrian-vehicle conflict and the more obvious the vehicle yielded to the pedestrians. With the beginning time of the green light, less and less samples stop, the time of the samples waiting on the roadside or before running became shorter and the behavior of starting running before entering the sidewalk or still running after leaving the crosswalk became more obvious.

Stepwise regression analysis was performed on 9 variables that discussed above, and only vehi_yield and beforeWait_time closely finally related to the delta-T to the end of the green.

Other variables are also closely related. In the samples that still ran after leaving the sidewalk, the women were more than men. If the running behavior was caused by pedestrian-vehicle conflict, the running time would be shorter, the pedestrians would tend to choose the path type 3 and type 4 , the behavior of looking around was more obvious, less samples would start running before enter the 
crosswalk or still run after leaving the sidewalk. The running time was relatively long if the vehicle yield to the pedestrian in the conflict. Car rather than bus was easier yielding to pedestrians in the conflict. The pedestrians with stopping behavior were more obvious with the behavior of looking around, and the latter would wait longer time on the roadside or before running. The longer the waiting time was on the roadside, the longer the waiting time before running.

\subsection{Limitations and Prospect}

There are some limitations in this study to be addressed in the future work. Firstly, the behavior of pedestrian is sometimes affected by the environment, such as long-distance vehicles, but it cannot be observed due to the limit of the camera range. Next, we made some simplified in the process of coding variables. For example, the behavior of looking at vehicles was measured by head movement, age was simplified into two groups. Finally, only objective factors were considered while psychological aspect is neglected. These limitations need further research in the future.

The findings above suggest several applications on development of intelligent warning system which is a compromise between traffic efficiency and pedestrian safety. Next, the research results can also be used to develop safety measures such as when to strengthen supervision and management. Besides, it can also be used to carry out a special education to the pedestrians and drivers. Moreover, this study can provide data and theoretical support for the further study.

\section{Acknowledgements}

This work is supported by the China National Natural Science Foundation (11302022) and the Fundamental Research Funds for the Central Universities (2015JBWY020).

\section{References}

[1]. J. Yang, W. Deng, J. Wang, et al. Modeling pedestrians' road crossing behavior in traffic system micro-simulation in China. Transportation Research Part A, 40, 280-290, (2006).

[2]. Information on: Automobiles, X., Pedestrian protection: Guangzhou Auto Honda establishes a safety benchmark, (2009). <http://www.auto.ifeng.com/news/domesticindustry/20090828/92508.shtml> (Retrieved 27.12.09).

[3]. CRTASR, China Road Traffic Accidents Statistics Report of 2004. In Traffic Administration Bureau of China State Security Ministry, Beijing, China, (2005)

[4]. C. X. Wu, L. Yao, K Zhang. The red-light running behavior of electric bike riders and cyclists at urban intersections in China: An observational study. Accident Analysis and Prevention 49 (2012) 186- 192.

[5]. X. L. Zhuang and C. X. Wu. The safety margin and perceived safety of pedestrians at unmarked roadway. Transportation Research Part F 15 (2012) 119-131.

[6]. H. C. Wang and C. J. Zheng. Red-light running behavior of pedestrians based on survival analysis. Journal of East China Jiaotong University, Vol. 32 No. 2 Apr., 2015.

[7]. J. Sun, Y. Yang, K. P. Li, et al. Wang, The virtual experiment of pedestrians crossing behavior at signalized intersection, Joural of Tongji University (Natural Science), V01.39 No.5, May.2011.

[8]. Z. Y. Sun, J. Rong, M. He et al. The gap acceptance of the pedestrian at the crosswalk of signalized intersection, Joural of Highway and Transportation Research and Development, Vol.21 No.11, 1002-0268 (2004)11-0102-03.

[9]. D. R. Geruschat, S. E. Hassan, and K. A. Turano. Gaze behavior while crossing complex intersections. Optometry and Vision Science, 80, 515-528 (2003). 
[10]. S. J. Murray. The effects of simulated cellular phone conversation on road-crossing safety. Thesis of Doctor of Philosophy, University of Canterbury (pp.237) , (2006)

[11]. T. Rosenbloom, A. Ben-Eliyahu, and D. Nemrodov. Children's crossing behavior with an accompanying adult. Safety Science, 46, 1248-1254., (2007)

[12]. D. Yagi. Beliefs, motives and situational factors related to pedestrians' self-reported behavior at signal-controlled crossings. Transportation Research Part F: Traffic Psychology and Behavior, 3, 1-13, (2000).

[13]. X. B. Yang, M. Huan, M. Abdel-Aty, et al. Y. Gao, A hazard-based duration model for analyzing crossing behavior of cyclists and electric bike riders at signalized intersections. Accident Analysis and Prevention 74 (2014) 33-41.

[14]. R. O. Phillips, T. Bjørnskau, R. Hagman, F. Sagberg. Reduction in car-bicycle conflict at a road-cycle path intersection: evidence of road user adaptation. Transportation Research Part F: Traffic Psychology and Behaviour 14 (2), 87-95. (2011) 